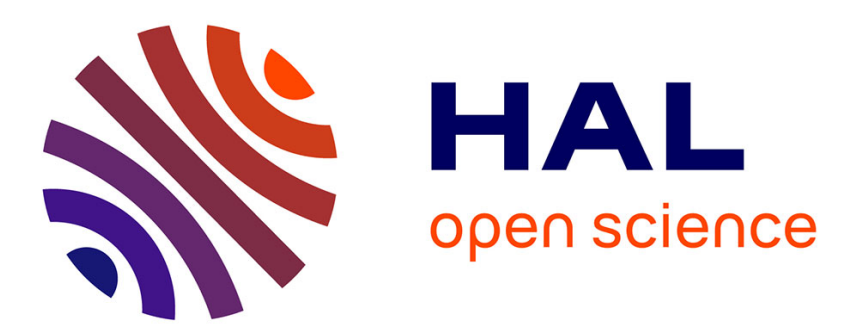

\title{
On the stability of sets defined by a finite number of equalities and inequalities
}

\author{
J. Frederic Bonnans, Geneviève Launay
}

\section{To cite this version:}

J. Frederic Bonnans, Geneviève Launay. On the stability of sets defined by a finite number of equalities and inequalities. [Research Report] RR-0738, INRIA. 1987. inria-00075814

\section{HAL Id: inria-00075814 \\ https://hal.inria.fr/inria-00075814}

Submitted on 24 May 2006

HAL is a multi-disciplinary open access archive for the deposit and dissemination of scientific research documents, whether they are published or not. The documents may come from teaching and research institutions in France or abroad, or from public or private research centers.
L'archive ouverte pluridisciplinaire HAL, est destinée au dépôt et à la diffusion de documents scientifiques de niveau recherche, publiés ou non, émanant des établissements d'enseignement et de recherche français ou étrangers, des laboratoires publics ou privés. 


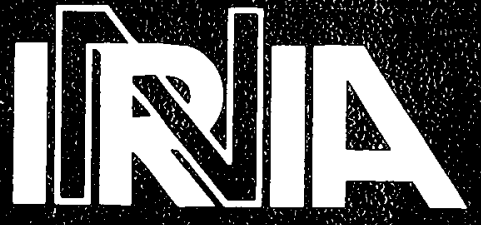

UNITÉ DE RECHERCHE INRIA-ROCOUENCOURT

Institert Nation. de Decherche enthrornation et en Automatique

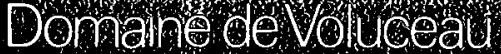

Rocolveneourt (1) 1.

7 - 1 (7)

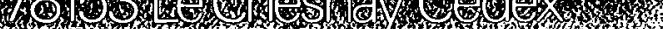

\section{Rapports de Recherche}

$$
\mathbf{N}^{\circ} 738
$$

\section{ON THE STABILITY OF SETS DEFINED BY A FINITE NUMBER OF EQUALITIES AND INEQUALITIES}

\section{Joseph Frédéric BONNANS}

Geneviève LAUNAY 
ON THE STABILITY OF SETS DEFINED BY A FINITE

NUMBER OF EQUALITIES AND INEQUALITIES

JOSEPH FREDERIC BONNANS" AND GENEVIEVE LAUNA ${ }^{\#}$

\begin{abstract}
Let a set be defined by a finite number of equalities and inequalities. For smooth data the condition of Mangasarian and Fromovitz is known to be equivalent to the local stability - in a strong sense - of the set. We study here weaker forms of stability. Namely, we state a condition generalizing the one of Mangasarian and Fromovitz that, for some weak form of. stability, is necessary. If the gradients of the equality constraints are linearly independent (or if then is no equality constraint) this condition is also sufficient.
\end{abstract}

Key words. Stability analysis, nonlinear constraints.

SUR LA STABILITE DES ENSEMBLES DEFINIS PAR UN NOMBRE FINI

D' EGALITES ET D' INEGALITES.

Résumé. Soit un ensemble décrit par un nombre fini d'égalités et d'inégalités. On sait que lorsque les données sont régulières, la condition de Mangasarian et Fromovitz équivaut à la stabilité locale -dans un sens fort- de l'ensemble. Nous étudions ici des formes plus faibles de stabilité. Nous énonçons une condition généralisant celle de Mangasarian et Fromovitz qui, pour une forme faible de stabilité, est nécessaire. Si les gradients des contraintes d'égalité sont linéairement indépendants (ou $s^{\prime}$ il $n^{\prime}$ y a pas de contraintes d'égalités) cette condition est aussi suffisante.

\#INRIA, Rocquencourt, 78153 Le Chesnay, France 
1. Introduction. We consider a subset of $\mathbb{R}^{n}$ ( $n$ being a positive integer) defined in the following way :

$$
D=\left\{x \in \mathbb{R}^{n} ; g(x)=0 \text { and } h(x) \leqq 0\right\} \text {, }
$$

where $g$ and $h$ are smooth mappings from $\mathbb{R}^{n}$ into $\mathbb{R}^{P}$ and $\mathbb{R}^{q}$, respectively; the vector order relation is taken componentwise. Let $\bar{x}$ be in $D$. Our problem is to study the stability of $D$ (near $\bar{x}$ ) when $g$ and $h$ are subject to small pertubations. As we are interested in a local analysis, we will suppose in all the paper that $h(\bar{x})=0$. Specifically we consider pertubations of the form

$$
D^{\varepsilon}=\left\{x \in \mathbb{R}^{n} ; g^{\varepsilon}(x)=0 \text { and } h^{\varepsilon}(x) \leq 0\right\}
$$

with $\varepsilon$ e $\mathbb{R}^{+}, g^{0}=g$ and $h^{0}=h$ and the mapping $(\varepsilon, x) \rightarrow\left(g^{\varepsilon}(x), h^{\varepsilon}(x)\right)$ smooth enough, and we call them smooth pertubations. The problem is to give an estimate of $\operatorname{dist}\left(\bar{x}, D^{\varepsilon}\right)$ defined by

$$
\operatorname{dist}\left(\bar{x}, D^{\varepsilon}\right)=\min \left\{\left\|\bar{x}-x^{\varepsilon}\right\| ; x^{\varepsilon} \text { e } D^{\varepsilon}\right\}
$$

with the help of the successive derivatives of $g$ and $h$ at $\bar{x}$.

Such problems have been already studied, mainly in connection with optimization problems. Linear systems of equalities and inequalities have been studied in J.W. Daniel [1]. The behavior of linear systems seems rather well understood. Nonlinear systems are studied in S.M. Robinson [3]. In particular, the result of [3] is that the condition of Mangasarian and Fromovitz [2], namely

$$
\begin{aligned}
& \nabla g(\bar{x}) \text { is surjective } \\
& \text { There exists } d \text { in } \mathbb{R}^{n} \text { such that } \\
& \nabla g(\bar{x})^{t} d=0 \text { and } \nabla h(\bar{x})^{t} d<0
\end{aligned}
$$

is necessary and sufficient for some strong form of stability. our aim is to study a weak form of stability, for which it is necessary to consider the second derivatives of the data. We state a new condition (which is a generalization of (MF)), necessary for weak stability. If $\nabla g(\bar{x})$ is surjective, this condition is also necessary. 
The paper is as follows. In section 2 we study a new concept of stability, the $k$-stability and we caracterise it on some simple examples. In section 3 we prove that the k-stability for $k<2$ implies the 1 -stability. In section 4 we establish a technical result concerning linear systems of equalities and inequalities. In section 5 , we give a necessary condition for 2-stability; we prove that if the gradients of the equality constraints are linearly independent, this condition is also sufficient for 2-stability. 
2. The concept of k-stability. We define the $k$-stability, relate it to the result of S.M. Robinson [2] and give a study of simple examples.

DEFINITION 1. The family of perturbations $D^{\varepsilon}$ is $\ell$-smooth ( $l$ positive integer) if the mapping $(\varepsilon, x) \rightarrow\left(g^{\varepsilon}(x), h^{\varepsilon}(x)\right)$ is $\ell$ times continuously differentiable.

DEFINITION 2. Let $\bar{x}$ be in $D$. We say that $D$ is $k$-stable at $\bar{x}(k>0$, real) if $g$ and $h$ are $c^{\ell}$ and, for any $\ell$-smooth pertubation $D^{\varepsilon}$ with $\ell \geqq k$, there exists $K>$ 0 such that

$$
\operatorname{dist}\left(\bar{x}, D^{\varepsilon}\right) \leqq K \varepsilon^{1 / k} \text {. }
$$

The simplest form of stability is obviously when $k=1$; in this case a complete characterization is known :

THEOREM 1 (S.M. Robinson, [2]). The set $D$ is 1 -stable at $\bar{x}$ iff condition (MF) holds. $\square$

It remains, however, to study the k-stability for $k>1$. In order to get some intuition of the kind of result we might prove, let us consider two simple cases. As the results are easily obtained we state them without proof.

a) The dimensions are $\mathrm{n}=\mathrm{p}=1$ and $\mathrm{q}=0$ and $\mathrm{g}$ is $\mathrm{C}^{\infty}$. Then :

- if all derivatives of $g$ are null at $\bar{x}$, there is no k-stability of $D$ at $\overline{\mathrm{x}}$.

- otherwise let $k_{0}$ be the index of the first non-null derivative of $g$. If $k_{0}$ is even, there is no k-stability of $D$ at $\bar{x}$. If $k_{0}$ is odd, then $D$ is $k_{0}$-stable.

b) The dimensions are $n=1, p=0, q=1$ and $h$ is $c^{\infty}$. Then:

- If all derivatives of $h$ are null, there is no k-stability of $D$ at $\bar{x}$.

- otherwise, let $k_{0}$ be the index of the first non-null derivative of $h$ at

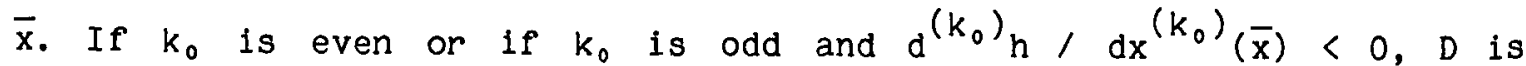
$k_{0}$-stable; otherwise there is no k-stability of $D$ at $\bar{x}$.

In view of these results, we may think that the integer values of $k$ are of special importance and that, for integer values of $k$, the k-stability is strongly related to the derivatives of $g$ and $h$ at $\bar{x}$, up to the order $k$. 
3. The k-stability for $1<\mathbf{k}<2$. The study of this case reduces to the following Theorem, showing again that integer values of $k$ are of special importance.

THEOREM 2. If $g$ and $h$ are $C^{2}$, the $k$-stability of $D$ at $\bar{x}$ for $k<2$ implies the 1-stability.

Before giving the proof, let us state some definitions and notations that will be useful in the sequel of this paper.

DEFINITION 3. A pertubation $D^{\varepsilon}$ of $D$ is said to be elementary if it is of the form

$$
\begin{aligned}
& g^{\varepsilon}(x)=g(x)-\varepsilon c, \\
& h^{\varepsilon}(x)=h(x)-\varepsilon c^{\prime},
\end{aligned}
$$

with $\left(c, c^{\prime}\right)$ given in $\mathbb{R}^{p} \times \mathbb{R}^{q}$.

Let $\left\{x^{\varepsilon}\right\}$ be a sequence in $\mathbb{I R}^{n}$ converging to $\bar{x}$ when $\varepsilon \rightarrow 0$. We notice that we may always write

$$
x^{\varepsilon}=\bar{x}+\alpha^{\varepsilon} d^{\varepsilon}
$$

with

$$
\begin{aligned}
& \alpha^{\varepsilon}=\left\|x^{\varepsilon}-\bar{x}\right\|, \\
& \left\|d^{\varepsilon}\right\|=1 .
\end{aligned}
$$

PROOF OF THEOREM 2. Let $D$ be $k$-stable at $\bar{x}$ with $k<2$. We consider an elementary pertubation of the above form. Then there exists $K>0$ and $\left\{x^{\varepsilon}\right\}$, with $x^{\varepsilon} \in D^{\varepsilon}$, such that $\left\|x^{\varepsilon}-\bar{x}\right\| \leqq K \varepsilon^{1 / k}$. This is equivalent, with the above notations, to $\alpha^{\varepsilon} \leqq K \varepsilon^{1 / k}$; hence $\left(\alpha^{\varepsilon}\right)^{2}=O(\varepsilon)$. As $x^{\varepsilon}=\bar{x}+\alpha^{\varepsilon} d^{\varepsilon}$ is in $D^{\varepsilon}$ and $(\mathrm{g}, \mathrm{h})$ are $\mathrm{C}^{2}$, we obtain

$$
\begin{aligned}
& g\left(x^{\varepsilon}\right)=\alpha^{\varepsilon} \nabla g(\bar{x})^{t} d^{\varepsilon}+o(\varepsilon)=\varepsilon c, \\
& h\left(x^{\varepsilon}\right)=\alpha^{\varepsilon} \nabla h(\bar{x})^{t} d^{\varepsilon}+o(\varepsilon) \leqq \varepsilon c^{\prime} .
\end{aligned}
$$


The relation on $g$ and the fact that $c$ is arbitrary imply that the range of $\nabla g(\bar{x})$ is dense in $\mathbb{R}^{p}$, hence (as any vector space of $\mathbb{R}^{p}$ is closed) $\nabla g(\bar{x})$ is surjective. Now suppose that $c=0$ and $c^{\prime}<0$ (componentwise). Let $r^{\varepsilon}$ be the minimum ( $(2)$ norm solution of

$$
\nabla g(\bar{x})^{t}\left(\alpha^{\varepsilon}{ }^{\varepsilon}+r^{\varepsilon}\right)=0
$$

As $\nabla_{g}(\bar{x})$ is surjective, $r^{\varepsilon}=O\left(\alpha^{\varepsilon} \nabla_{g}(\bar{x})^{t} d^{\varepsilon}\right)=O(\varepsilon)$, hence $y^{\varepsilon}=\varepsilon^{-1}\left(\alpha^{\varepsilon} d^{\varepsilon}+r^{\varepsilon}\right)$ satisfies

$$
\begin{aligned}
& \nabla g(\bar{x})^{t} y^{\varepsilon}=0, \\
& \nabla h(\bar{x})^{t} y^{\varepsilon} \leqq c^{\prime}+o(\varepsilon) / \varepsilon .
\end{aligned}
$$

The right hand side of the above inequality is strictly negative for $\varepsilon$ small enough. This proves that (MF) holds; hence, by Theorem 1, D is 1-stable at $\bar{x}$. 口 
4. Some results concerning linear systems. Before going on with the study of the 2-stability, we have to state some technical results concerning linear systems of equalities and inequalities. Consider the system

$$
A z=e, B z \leqq f,
$$

with $A, B$ being matrices and $z$, e, $f$ vectors of convenient (finite) dimensions.

Denote

$$
\begin{aligned}
& B_{i}: \text { the ith line of } B, \\
& B_{J}=\left\{B_{i} ; i \in J\right\} \\
& f_{J}=\left\{f_{i} ; i \in J\right\} .
\end{aligned}
$$

We will have to consider the associated homogeneous system

$$
A z=0, \quad B z \leq 0
$$

Define

$$
\begin{aligned}
& I=\left\{i ; \text { there exists } z^{i} \text { satisfying }(L H) \text { and } B_{i} z^{i}<0\right\} \\
& \bar{I}=\left\{i ; \text { there exists no } z \text { satisfying }(L H) \text { and } B_{i} z<0\right\}
\end{aligned}
$$

We denote $f^{+}=\max (f, 0)$ and $f^{-}=\min (f, 0)$.

The aim of this section is to prove the following result.

THEOREM 3. There exists $M>0$ such that, for any $(e, f)$ such that ( $L$ ) has a solution, then (L) has a solution $z$ satisfying

$$
\|z\| \leqq M\left(\| \text { e }\|+\| \mathrm{f}_{\bar{I}}^{+}\|+\| \mathrm{f}_{\mathrm{I}^{-}}^{-} \|\right)
$$

For clarity we state two preliminary Lemmas. Our Lemma 2 is a corollary of Lemma 3.5 of J.W. Daniel [1] ; we give a proof (different from the one of [1]) for the convenience of the reader. 
LEMMA 1. If $z$ satisfies

$$
A z=0, B \overline{\bar{I}} z \leqq 0,
$$

then $B_{\bar{I}} z=0 . \quad \square$

PROOF. Let $z^{i}$ be as in the definition of I. Define $z^{0}=\sum_{i \in I} z^{i}$. Then obviously $A z^{0}=0, B_{I} z^{0}<0, B_{\bar{I}} z^{0} \leqq 0$.

If Lemma 1 does not hold, there exists $j \in \bar{I}$ and $w$ such that

$$
A W=0, \quad B_{\bar{I}} W \leqq 0, \quad B_{j} w<0 .
$$

Then, for $\alpha>0$ large enough, $z=w+\alpha z^{0}$ satisfies (LH) and $B_{j} z<0$; hence jeI, in contradiction with the definition of $I$.

LEMMA 2. There exists $M_{1}>0$ such that any solution $(z, e, f)$ of

$$
A z=e, \quad B_{\bar{I}} z \leqq f_{\bar{I}},
$$

satisfies

$$
\left\|B_{\bar{I}} z\right\| \leqq M_{1}\left(\| \text { e }\|+\| \mathrm{f}_{\bar{I}}^{+} \|\right) \text {. }
$$

PROOF. If the conclusion is false, there exists $\left\{\left(z^{n}, e^{n}, f^{n}\right)\right\}$ satisfying ( $\left.\bar{L}\right)$ and

$$
\left\|B_{\bar{I}} z^{n}\right\|>n\left(\left\|e^{n}\right\|+\left\|\left(f_{\bar{I}}^{n}\right)^{+}\right\|\right)
$$

Normalizing the sequence if necessary, we may suppose that $\left\|B_{\bar{I}} z^{n}\right\|=1$; hence $e^{n} \rightarrow 0$ and $\left(f_{\bar{I}}^{n}\right)^{+} \rightarrow 0$. As $A z^{n}=e^{n}$ and $B \bar{I}^{n}$ are bounded, there exists a bounded sequence $\bar{Z}^{n}$ with $A \bar{z}^{-n}=A z^{n^{\prime}}=e^{n}$ and $B_{\bar{I}} \bar{z}^{-n}=B_{\bar{I}^{2}} z^{n}\left(\hat{f}_{\bar{I}}^{n}\right)^{+}$. Extracting a subsequence if necessary we may suppose that $\bar{z}^{n} \rightarrow \bar{z}$. Passing to the limit we obtain

$$
\left\|B_{\bar{I}} \bar{z}\right\|=1, \quad A \quad \bar{z}=0, \quad B_{\bar{I}} \bar{z} \leqq 0,
$$


in contradiction with Lemma 1.

$\square$

PROOF OF THEOREM 3. Let $(z, e, f)$ satisfy ( $L$ ). From Lemma 2 we deduce that

$$
\|A z\|+\left\|B_{\bar{I}} z\right\| \leqq\left(M_{1}+1\right)\left(\|e\|+\left\|f_{\bar{I}^{+}}^{+}\right\|\right) \text {. }
$$

Hence there exists $M_{2}>0$ independent of $(z, e, f)$ such that there exists $\bar{z}$ satisfying

and

$$
A \bar{z}=A z, \quad B_{\bar{I}} \bar{z}=B_{\bar{I}} z,
$$

$\|\bar{z}\| \leqq M_{2}\left(\|\right.$ e $\left.\|+\| \mathrm{f}_{\bar{I}}^{+} \|\right)$.

Let $z^{0}$ be as in the proof of Lemma 1. Then $z^{\alpha}=\bar{z}+\alpha z^{0}$ satisfies, for any $\alpha \geqq 0$

$$
A z^{\alpha}=e, \quad B_{\bar{I}} z^{\alpha} \leqq f_{\bar{I}},
$$

and the inequality $B_{I} z^{\alpha} \leqq f_{I}$ will be satisfied iff (as $B_{i} z^{0}<0$ )

$\alpha \geqq\left(B_{i} \bar{z}-f_{i}\right) / B_{i} z^{0}, \quad \forall i \in I$.

Denote $\beta=\min \left\{\left|B_{i} z^{0}\right|\right.$, i $\left.\in I\right\} ;$ the above inequality will be satisfied if

$$
\alpha \geqq \beta^{-1}\left(\left|B_{i} \bar{z}\right|+f_{i}^{+}\right)
$$

and this with the above estimate on $\bar{z}$ proves the result. 
5. Study of the 2-stability. The reduction of the k-stability to the 1-stability for $k<2$ does not hold for $k=2$. Take for instance $n=2, p=1$, $\mathrm{q}=0$ and $\mathrm{g}(\mathrm{x})=\left(\mathrm{x}_{1}\right)^{2}-\left(\mathrm{x}_{2}\right)^{2}$.

For any element $y$ of $\mathbb{R}^{n}$, we denote by $y^{t} \nabla^{2} g(\bar{x})$ y the vector of $\mathbb{R}^{p}$ whose $i^{\text {th }}$ component is $y^{t} \nabla^{2} g_{i}(\bar{x}) y$. Let us state the following condition :

For any $\left(c, c^{\prime}\right)$ in $\mathbb{R}^{p} \times \mathbb{R}^{q}$, there exists $(y, z)$ in $\mathbb{R}^{n} \times \mathbb{R}^{n}$ satisfying

(i) $\quad \nabla g(\bar{x})^{t} y=0, \quad \nabla h(\bar{x})^{t} y \leq 0$,

(ii) $\nabla g(\bar{x})^{t} z+\frac{1}{2} y^{t} \nabla^{2} g(\bar{x}) y=c$,

(iii) For any $i$ in $\mathbb{R}^{q}$, either $\nabla h_{i}(\bar{x})^{t} y<0$ or

$$
\nabla h_{i}(\bar{x})^{t} z+\frac{1}{2} y^{t} \nabla^{2} h_{i}(\bar{x}) y \leqq c_{i}^{\prime} \cdot
$$

This new condition is a generalization of (MF) because, if in (BL) we may always take $\mathrm{y}=0$, then the above relations reduce to

$$
\nabla g(\bar{x})^{t} z=c, \quad \nabla h(\bar{x})^{t} z \leqq c^{\prime}
$$

for any ( $\left.c, c^{\prime}\right)$, which is easily seen to be equivalent to (MF). We will prove the following result :

THEOREM 4. If $D$ is 2-stable at $\bar{x}$, then condition (BL) holds.

However, we will obtain it as a particular case of a more general result, involving uniform estimates with respect to a set of constants $\left(c, c^{\prime}\right)$.

DEFINITION 4. Let $\tau$ be a subset of $\mathbb{R}^{p} \times \mathbb{R}^{q}$. We say that $D$ is uniformly $k$-stable at $\bar{x}$ with respect to $\tau$ if there exists $K>0$ such that, for any $\left(c, c^{\prime}\right)$ in $\tau$, there exists a path $\left\{x^{\varepsilon}\right\}$ in the elementary pertubation $D^{\varepsilon}$ associated to (c, c') with

$$
\left\|\bar{x}-x^{\varepsilon}\right\| \leqq K|\varepsilon|^{1 / k}\left(\|c\|+\left\|c^{\prime}\right\|\right)^{1 / k} \text {. }
$$


As an example, take $\tau=\left\{\left(\begin{array}{llll}\alpha & c, \alpha c^{\prime}\end{array}\right), \alpha \in \mathbb{R}\right\}$. If $D$ is k-stable at $\bar{x}$, the constant associated to $\left(c, c^{\prime}\right)$ being $K$, then $D$ is uniformly $k$-stable at $\bar{x}$ with respect to $\tau$, with the same constant $K$ (this is a justification of the power $1 / k$ associated to $\|c\|+\left\|c^{\prime}\right\|$ in definition 4$)$. We will prove the following result :

THEOREM 5. Let $D$ be uniformly 2-stable at $\bar{x}$ with respect to $\tau$. Then there exists $K_{1}>0$ such that to each $\left(c, c^{\prime}\right.$ ) in $\tau$ is associated $(y, z)$ satisfying (BL) and

$$
\|z\|+\|y\|^{2} \leqq K_{1}\left(\|c\|+\left\|c^{\prime}\right\|\right)
$$

PROOF. We consider the elementary pertubation associated to an element (c, $\left.c^{\prime}\right)$ of $\tau$. Let $x^{\varepsilon}$ be an element of $D^{\varepsilon}$ satisfying

$$
\left\|x^{\varepsilon}-\bar{x}\right\| \leqq K|\varepsilon|^{1 / 2}\left(\|c\|+\left\|c^{\prime}\right\|\right)^{1 / 2} \text {. }
$$

Writing, as in section $3, x^{\varepsilon}=\bar{x}+\alpha^{\varepsilon} d^{\varepsilon}$, we obtain

$$
\begin{aligned}
& g\left(x^{\varepsilon}\right)=\alpha^{\varepsilon} \nabla g(\bar{x})^{t} d^{\varepsilon}+\frac{\left(\alpha^{\varepsilon}\right)^{2}}{2}\left(d^{\varepsilon}\right)^{t} \nabla^{2} g(\bar{x}) d^{\varepsilon}+o(\varepsilon)=\varepsilon c, \\
& h\left(x^{\varepsilon}\right)=\alpha^{\varepsilon} \nabla h(\bar{x})^{t} d^{\varepsilon}+\frac{\left(\alpha^{\varepsilon}\right)^{2}}{2}\left(d^{\varepsilon}\right)^{t} \nabla^{2} h(\bar{x}) d^{\varepsilon}+o(\varepsilon) \leqq \varepsilon c^{\prime} \cdot
\end{aligned}
$$

Case 1 : An extracted sequence of $\alpha^{\varepsilon} / \varepsilon$ has a limit point $\gamma$. Dividing the above system by $\varepsilon$ and passing to the limit for the extracted sequence, we obtain (d being a limit-point of $\left\{d^{\varepsilon}\right\}$ ):

$$
\gamma \nabla_{g}(\bar{x})^{t} d=c, \quad \gamma \nabla h(\bar{x})^{t} d \leqq c^{\prime}
$$

Then statements (i) - (iii) of (BL) are satisfied with $z=\gamma d, y=0$. Applying Theorem 3 to the linear system $\nabla g(\bar{x})^{t} z=c, \nabla h(\bar{x})^{t} z \leqq c^{\prime}$ we see that (BL) may be satisfied with $y=0$ and $\|z\| \leqq M\left(\|c\|+\left\|c^{\prime}\right\|\right)$, the constant $M$ depending only on $\nabla g(\bar{x})$ and $\nabla h(\bar{x})$ (not on (c, $\left.c^{\prime}\right)$ ). 
Case $2:$ As $\varepsilon \rightarrow 0, \alpha^{\varepsilon} / \varepsilon \rightarrow+\infty$. Dividing the above quadratic system by $\alpha^{\varepsilon}$ and passing to the limit, $d$ being again a limit-point of $d^{\varepsilon}$, we obtain

$$
\nabla g(\bar{x})^{t} d=0, \quad \nabla h(\bar{x})^{t} d \leq 0
$$

Put $d^{\varepsilon}=d+\beta^{\varepsilon} v^{\varepsilon}$ with $B^{\varepsilon}=\left\|d^{\varepsilon}-d\right\|$. We expand here $d^{\varepsilon}$ in the same way we did for $x^{\varepsilon}$. For an extracted sequence, $d^{\varepsilon} \rightarrow d$, hence $\beta^{\varepsilon} \rightarrow 0$ and $\left(\alpha^{\varepsilon}\right)^{2} \beta^{\varepsilon}=o(\varepsilon)$ because of the 2-stability. Hence, for this extracted sequence, and using the relations on $d$ :

$$
\alpha^{\varepsilon} \beta^{\varepsilon} \nabla g(\bar{x})^{t} v^{\varepsilon}+\frac{\left(\alpha^{\varepsilon}\right)^{2}}{2} d^{t} \nabla^{2} g(\bar{x}) d=\varepsilon c+o(\varepsilon),
$$

and if $\nabla h_{i}(\bar{x})^{t} d=0$, then

$$
\alpha^{\varepsilon} \beta^{\varepsilon} \nabla h(\bar{x})^{t} v^{\varepsilon}+\frac{\left(\alpha^{\varepsilon}\right)^{2}}{2} d^{t} \nabla^{2} h(\bar{x}) d \leq \varepsilon c^{\prime}+o(\varepsilon) .
$$

Dividing the above relations by $\varepsilon$ and defining

$$
\begin{aligned}
& y^{\varepsilon}=\varepsilon^{-y / 2} \alpha^{\varepsilon} d, \\
& z^{\varepsilon}=\varepsilon^{-1} \alpha^{\varepsilon} \beta^{\varepsilon} v^{\varepsilon},
\end{aligned}
$$

we get

$$
\nabla g(\bar{x})^{t} z^{\varepsilon}+\frac{1}{2}\left(y^{\varepsilon}\right)^{t} \nabla^{2} g(\bar{x}) y^{\varepsilon}=c+o(\varepsilon) / \varepsilon,
$$

and if $\nabla h_{i}(\bar{x})^{t} y^{\varepsilon}=0$ then

$$
\nabla h_{i}(\bar{x})^{t} z^{\varepsilon}+\frac{1}{2}\left(y^{\varepsilon}\right)^{t} \nabla^{2} h_{i}(\bar{x}) y^{\varepsilon} \leq c^{\prime}+o(\varepsilon) / \varepsilon \text {. }
$$

As $|\varepsilon|^{-1 / 2} \alpha^{\varepsilon} \leq K\left(\|c\|+\left\|c^{\prime}\right\|\right)^{1 / 2}, y^{\varepsilon}$ is bounded and converges for some subsequence to some $y=\gamma d$ with $0 \leqq \gamma \leqq K\left(\|c\|+\left\|c^{\prime}\right\|\right)^{1 / 2}$; hence

$$
\|y\|^{2} \leq(K)^{2}\left(\|c\|+\left\|c^{\prime}\right\|\right),
$$

and y satisfies (BLi). 
As $\mathrm{y}^{\varepsilon}$ is bounded we may, using Theorem 3, suppose that $\mathrm{z}^{\varepsilon}$ is also bounded and (extracting if necessary a new subsequence) converges towards some $z ;(y, z)$ satisfy (BLii - iii) and, applying Theorem 3 as in case 1 we get ( $K_{2}$ depending on $\nabla^{2} g(\bar{x})$ and $\left.\nabla^{2} h(\bar{x})\right)$ :

$$
\begin{aligned}
\|z\| & \leqq M\left(\|c\|+\left\|c^{\prime}\right\|+K_{2}\|y\|^{2}\right) \\
& \leqq\left(M+(K)^{2} K_{2}\right)\left(\|c\|+\left\|c^{\prime}\right\|\right),
\end{aligned}
$$

which proves the Theorem. $\quad$

We conjecture that condition (BL) - or perhaps the strenghtened condition obtained by supposing that $(y, z)$ satisfy the estimate of the conclusion of Theorem 5, with $\tau=\mathbb{R}^{p} \times \mathbb{R}^{q}$ - is sufficient for 2-stability. This seems not easy to prove, however; the main difficulty is due to the lack of knowledge about the behaviour of the solution of quadratic systems. Nevertheless, we can state the following partial converse result.

THEOREM 6. Let $\bar{x}$ be a point of $D$ at which condition (BL) holds. If in addition $g$ and $h$ are $C^{3}$ and $\nabla g(\bar{x})$ is surjective (or no equality constraint is present), then $\mathrm{D}$ is 2 -stable at $\overline{\mathrm{x}}$.

PROOF. Let $D^{\varepsilon}$ be a smooth pertubation of $D$ associated to mappings $g^{\varepsilon}$ and $h^{\varepsilon}$. We shall construct a point $x^{\varepsilon}$ in $D^{\varepsilon}$ sufficiently close to $\bar{x}$. Let us denote $c=$ $-\left.\frac{d}{d \varepsilon} g^{\varepsilon}(\bar{x})\right|_{\varepsilon=0}$ and define $c^{\prime}$ by

$$
c_{i}^{\prime}=\min \left(0,-\frac{d}{d \varepsilon} h_{i}^{\varepsilon}(\bar{x})_{\varepsilon=0}\right)-1
$$

Let $(y, z)$ be associated to $\left(c, c^{\prime}\right)$ by condition $(B L)$. We shall choose $x^{\varepsilon}$ of the form

$$
\mathrm{x}^{\varepsilon}=\bar{x}+\varepsilon^{y / 2} y+\varepsilon z+r^{\varepsilon}
$$

As $g$ is $C^{3}$ (and $(\varepsilon, x)+g^{\varepsilon}(x)$ is $C^{2}$ ), we find, using (BL), that 


$$
\begin{aligned}
g^{\varepsilon}\left(\bar{x}+\varepsilon^{1 / 2} y+\varepsilon z\right) & =g\left(\bar{x}+\varepsilon^{1 / 2} y+\varepsilon z\right)-\varepsilon c+O\left(\varepsilon^{2}\right) \\
& =\nabla g(\bar{x})^{t}\left(\varepsilon^{1 / 2} y+\varepsilon z\right)+\frac{\varepsilon}{2} y^{t} \nabla^{2} g(\bar{x}) y-\varepsilon c+O\left(\varepsilon^{3 / 2}\right) \\
& =O\left(\varepsilon^{3 / 2}\right) .
\end{aligned}
$$

As $\nabla g(\bar{x})$ is surjective, for $\varepsilon$ small enough, we may find $r^{\varepsilon}$ such that $r^{\varepsilon}=O\left(\varepsilon^{3 / 2}\right.$ ) and $x^{\varepsilon}=\bar{x}+\varepsilon^{1 / 2} y+\varepsilon z+r^{\varepsilon}$ satisfies (for $\varepsilon$ small enough):

$$
\begin{aligned}
& g^{\varepsilon}\left(x^{\varepsilon}\right)=0, \\
& \left\|x^{\varepsilon}-\bar{x}\right\| \leqq 2\|y\| \varepsilon^{y / 2} .
\end{aligned}
$$

Now consider the inequality constraints : we have

$$
h^{\varepsilon}\left(x^{\varepsilon}\right)=\nabla h(\bar{x})^{t}\left(\varepsilon^{1 / 2} y+\varepsilon z\right)+\frac{\varepsilon}{2} y^{t} \nabla^{2} h(\bar{x}) y+\varepsilon \frac{d}{d \varepsilon} h^{\varepsilon}(\bar{x}) \mid \varepsilon=0+O\left(\varepsilon^{3 / 2}\right) .
$$

If, for some $i, \nabla h_{i}(\bar{x})^{t} y<0$, this implies that $h_{i}^{\varepsilon}\left(x^{\varepsilon}\right)<0$ for $\varepsilon$ small enough. Otherwise (BL) implies that $\nabla h_{i}(\bar{x})^{t} y=0$; our choice of $c^{\prime}$ implies that

$$
h_{i}^{\varepsilon}\left(x^{\varepsilon}\right) \leqq-\varepsilon+O\left(\varepsilon^{3 / 2}\right) \text {, }
$$

which implies that the constraints are satisfied for $\varepsilon$ small enough. 
REFERENCES

[1] J.W. Daniel, on perturbations in systems of linear inequalities, SIAM J. on Numerical Analysis 10 (1973), 299-307.

[2] O.L. Mangasarian, S. Fromovitz, The Fritz John necessary optimality conditions in the presence of equality and inequality constraints. J. of Mathematical Analysis \& Applications 17 (1967), 37-47.

[3] S.M. Robinson, Stability theory for systems of inequalities, part II : differentiable nonlinear systems. SIAM J. on Numerical Analysis 13 (1976), 497-513. 
